\title{
Complementary analysis techniques applied on optimizing suspensions of yttria stabilized zirconia.
}

Michela Della Negra ${ }^{1}$, Søren Preben Vagn Foghmoes, Trine Klemens $\varnothing^{2}$.

Department of Energy Conversion and Storage, Technical University of Denmark, Frederiksborgvej 399, 4000 Roskilde, Denmark.

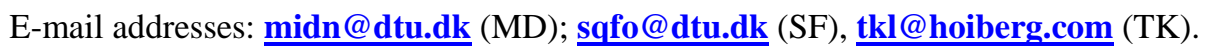

\section{Abstract}

Three different polymers with different functional groups and similar molecular weight were tested as dispersing agents for suspensions of yttria stabilized zirconia in ethanol: polyvinyl pyrrolidone, polyethylene imine, polyvinyl butyral/acetale. The stability of the system was assessed considering, in details, all the processing steps, including suspension de-agglomeration, slurry manipulation, quality of sintered tapes microstructure, and final layer leak tightness. Different analytical techniques were used to monitor ceramic deagglomeration and stability as a function of time, for different types of dispersing agent and to optimize the dispersants concentration: Electrokinetic Sonic Amplitude was used to obtain zeta potential, Multiple Light Scattering for evaluating sedimentation rate, and multi-wavelength laser light scattering for measuring particle size distribution. All the

\footnotetext{
${ }^{1}$ Corresponding author at Department of Energy Conversion and Storage, Technical University of Denmark, Frederiksborgvej 399, 4000 Roskilde, Denmark. Tel +45 46775852. Fax +45 46775858. Email address: midn@dtu.dk.

${ }^{2}$ Present address: Høiberg A/S, St. Kongensgade 59 A, 1264 Copenhagen K, Denmark
} 
results agree upon excellent performance of polyvinyl pyrrolidone and polyethylene imine as dispersing agents. The stability and dispersing power were finally utilized for preparing concentrated suspensions for tape casting and subsequently to sinter the tapes into dense ceramic pieces.

\section{Keywords}

Suspension (A); Tape casting (A); ZrO2 (D); Fuel Cells (E).

\section{Introduction}

Yttria stabilized zirconias (YSZs) is a widely used material for solid oxide fuel cells, solid oxide electrolysis cells, sensors and sintering substrates. The high ionic conductivity of 8YSZ ( $8 \mathrm{~mol} \% \mathrm{Y}_{2} \mathrm{O}_{3}$ doped $\mathrm{ZrO}_{2}$, called fully stabilized zirconia) makes it the preferred material for electrolytes [1], while the high mechanical strength and fracture toughness of zirconia containing meta stable tetragonal phases, e.g. $3 \mathrm{YSZ}\left(3 \mathrm{~mol} \% \mathrm{Y}_{2} \mathrm{O}_{3}\right.$ doped $\mathrm{ZrO}_{2}$, called partially stabilized zirconia) makes it advantageous for support layers [2].

For planar cells containing these materials, large, uniform and thin layers with controlled thickness are commonly produced by tape casting. This shaping technique is also industrial relevant due to the high throughput and the possibility to scale up to production line. In tape casting, the powders are dispersed in a solvent together with various organics (e.g. dispersant, binder, plasticizer) constituting a slurry that is cast into a thin sheet [3]. Organic solvent mixtures containing ketone or toluene in combination with ethanol are conventionally used as dispersing media due to the possibility of fine tuning their dielectric 
constant, vapor pressure and surface tension, in order to appropriately dissolve the organic compounds added in the slurry, wet the carrier and dry relatively quickly during production [4-6]. However, their use is also related with workspace health concerns. In this context, efforts are made to find alternatives that can improve the quality of the working environment, and also comply with the legislation requirements for making the production more environmentally friendly. A suitable alternative to the typical azeotropic mixtures of MEKEt (methyl ethyl ketone/ethanol) and toluene/ethanol could be ethanol. Even though pure ethanol is flammable and requires ATEX protection, the toxicity of the dispersing medium is reduced compared to the above mentioned azeotropic mixtures and the drying duration for the green body is still short and suitable for production lines [7-9]. A proper comminution and stabilization of the ceramic powder in the organic solvents is normally achieved by adding dispersing agents in the milling stage. The slurry stabilization is critical to several processing steps from comminution to sintering: it insures the reduction of the ceramic particle agglomerates in suspension to the point where complete densification can be achieved during sintering of the green body; it fosters also higher densification at the same sintering temperature, reproducibility and control of the final microstructure, both when fabricating dense layers or layers with tailored porosity. At the same time, a well disperse slurry is homogeneous, displays good flow at high ceramic solid loading, reducing issues correlated to excessive amount of solvent and consequent solvent evaporation.

The intent of this paper is to assess the efficiency of different polymeric dispersants for stabilizing 3YSZ particles in high solid-load ethanol suspensions, with the aim of producing reliable slurries for tape casting in a more environmental friendly solvent. As it 
will be shown later in the paper, the electrostatic repulsions between YSZ particles in suspension are not sufficient to ensure full dispersion stability. The performance of the polymeric dispersants is evaluated from the stage of milling to the final tape microstructure. Results from different analysis techniques on the colloidal suspensions give complementary information regarding size, charge, sedimentation and packing of the 3YSZ particles. The degree of compaction of the ceramic particles from suspension is used as an indication of good dispersion and densification properties [10]. The data are also compared to the behaviour of 3YSZ milled in ethanol without dispersant and the results help to understand the interactions between the ceramic powder, the solvent and different types of dispersing molecules.

Electrokinetic Sonic Amplitude (ESA) for deducting dynamic mobility (and thereby zeta potential) and Multiple Light Scattering (MLS) for evaluating sedimentation rate, are techniques appropriate for investigating suspensions with high ceramic load, which are necessary for shaping methods like tape casting. On the other hand the particle size distribution (PSD) is measured by multi-wavelength laser light scattering, which has to be carried out in diluted systems. The candidates performing satisfactorily in the stability study are implemented in slurries, which are tape casted. The properties of the final sintered film are studied by scanning electron microscopy (SEM) to verify the efficiency of the selected polymers in the entire process.

The investigated dispersants were polyvinyl pyrrolidone (PVP), polyethylene imine (PEI) and a polyvinyl butyral/acetal. For simplicity in the text, we will refer to this polymer as PVBAcetal. In addition to the obvious solubility requirement, our choice of dispersants was based on literature results and the general following considerations: PVP is a common 
dispersant used for thin film production in MEKEt, e.g. for LSM/YSZ tapes [11] and YSZ [4]; PEI is quoted several times as dispersant for alumina and zirconia powders in ethanol, especially for electrophoretic deposition [12-13]; PVBAcetal has a composition very close to the typical binders used in slurries for tape casting in organic solvents, and thereby potentially excludes incompatibility issues between organic molecules. Examples of PVB used with dual-role as dispersant and binder for tape casting in organic solvents can be found in [14-16].

The outcome of the work consists in the selection of two appropriate dispersants for 3YSZ suspensions in ethanol, which ensure proper ceramic comminution and stability, ideal processing conditions, allowing the production of dense and uniform sintered layers. The optimum amount of dispersants to be added in the slurry formulation was identified. We proved also that the tests conducted in suspensions of medium solid load are suitable models for the study of a tape casting system.

\section{Experiment}

\subsection{Materials and methods}

The starting powder was a commercial 3YSZ from Tosoh Co. (Tokyo, Japan). Declared crystallite size from the supplier: $290 \AA$ A. The specific surface area was measured by nitrogen gas-adsorption after degassing at $300^{\circ} \mathrm{C}$ using an Autosorp 1MP instrument

(Quantachrome Instruments, FL) and the value obtained was $13.47 \mathrm{~m}^{2} / \mathrm{g}$. The powder was kept at $120^{\circ} \mathrm{C}$ before suspension preparation to reduce water adsorption.

Branched PEI was purchased from Sigma-Aldrich. The molecular weight was determined by the supplier being circa $10^{\prime} 000$ by Gel Permeation Chromatography and $25^{\prime} 000$ by 
Liquid Chromatography. PVP with average molecular weight of 10’000 was also purchased from Sigma-Aldrich. Pioloform BL16 from Kuraray is a polyvinyl acetal produced reacting polyvinyl alcohol with butyraldehyde and acetaldehyde producing the PVBAcetal. The declared content of vinyl alcohol groups is $14-18 \mathrm{wt} \%$ and the amount of acetate groups is $0-4 \mathrm{wt} \%$. The molecular weight ranges from $18^{\prime} 000$ to $28^{\prime} 000 \mathrm{~g} / \mathrm{mol}$ and the glass transition temperature is $84^{\circ} \mathrm{C}$ [17].

All the suspensions were prepared in 99.9 vol\% absolute ethanol (CCS Healthcare AB, Borlänge, Sweden) with a ceramic load of $28.6 \mathrm{wt} \%$ in $1 \mathrm{~L}$ polyethylene bottles (100 g of 3YSZ in $250 \mathrm{~g}$ of ethanol). It was chosen to use intermediate YSZ solid load, as the YSZ powder in the control-suspension (YSZ in ethanol without dispersant) at $66 \mathrm{wt} \%$ solid load could not be wetted by the solvent, resulting in an impracticable experiment. A 3YSZ suspension was used to study the behaviour of the ceramic material in ethanol under rolling (without milling beads) for 15 days at $110 \mathrm{rpm}$. The suspensions for dispersant evaluation were milled for 2 days with $1 \mathrm{~kg}$ of 3/8" YSZ milling beads at a rotation speed of $220 \mathrm{rpm}$. The dispersant concentration was between 0 and 8 dry weight basis (dwb)\% for PEI, between 0 and $10 \mathrm{dwb} \%$ for PVP and between 0 and $16 \mathrm{dwb} \%$ for PVBAcetal. Dry weight bases indicates the ratio between the dry amount of dispersant and the amount of ceramic powder in suspension. Particle size distribution, sedimentation and zeta potential were measured on each sample almost simultaneously. To evaluate the comminution kinetic and the optimum milling time, a series of PSD measurements were performed on a YSZ suspension with $2 \mathrm{dwb} \%$ PEI as a function of milling time.

Some of the samples were duplicated in order to evaluate the variations due to sample preparation and handling procedures; measurements were repeated in order to evaluate the 
uncertainty of the analytical techniques. The sample duplications showed that the uncertainty related to sample preparation and handling is often dominating over the reproducibility of the analytical techniques.

On the basis of the performance in the stability tests, slurries stabilized with PEI and PVP were prepared for tape casting with a PVB binder. In this case, the ceramic solid load in ethanol was $66 \mathrm{wt} \%$, and the ratio between dispersant mass and YSZ mass was based on the optimum conditions determined in the stability tests. After milling for 3 days with $1 \mathrm{~kg}$ of 3/8" YSZ milling beads at a rotation speed of $220 \mathrm{rpm}$ the result of the comminution and dispersion were verified measuring particle size distribution. After binder addition the slurries rheological properties were tested with flow curves at controlled share rate using a Haake RheoStress 600 rheometer (Thermo Electron $\mathrm{GmbH}$ ) with parallel plate geometry and $0.8 \mathrm{~mm}$ gap. The slurries viscosity was adjusted in order to obtain $(3000 \pm 500) \mathrm{mPa} \cdot \mathrm{s}$ at shear rate $10 \mathrm{~s}^{-1}$. The slurries were filtered using a $37 \mu \mathrm{m}$ filter and de-aired prior to tape casting. A double doctor-blade system set at the casting height of $1100 \mu \mathrm{m}$ was used for the purpose. After drying, the green bodies were $180-210 \mu \mathrm{m}$ thick, and were laminated to increase thickness and facilitate handling. Samples were punched out from the laminates, and sintered above $1300^{\circ} \mathrm{C}$. The gas tightness of the sintered samples was leak tested with a vacuum technique as described in [18] to verify appropriate densification of the product. Polished cross-sections of the sintered samples were prepared and the grain boundaries made visible by thermally etching the samples at $65^{\circ} \mathrm{C}$ below sintering temperature for 2 hours. The microstructures were studied using a field-emission scanning electron microscope of the type Merlin FE-SEM, Carl Zeiss, Germany. 


\subsection{Analysis techniques}

The particle size distribution (PSD) analyses were performed with a laser diffractometer LD 13320 from Beckman-Coulter (Krefeld, Germany) with enhanced sub-micron measuring range using Polarization Intensity Differential Scattering (PIDS). All the samples were measured immediately after the milling stages. Each sample was measured in triplicates, every time by repeating the procedure from the sampling step. Measurements were also repeated with increasing obscuration, in order to make sure to detect small populations of large particles besides larger population of sub-micron particles [19]. Zeta potential of the YSZ suspensions was determined measuring the Electrokinetic Sonic Amplitude (ESA) with the instrument ZetaProbe of Colloidal Dynamic LLC (North Attleboro, USA). The mathematical relation between ESA and dynamic mobility is expressed in equation (1) [20]:

$$
E S A=A(\omega) \frac{z_{s} z_{g}}{z_{S}+z_{g}} \phi \frac{\rho_{p} \rho}{\rho} \mu_{D}
$$

Where $\mathrm{A}(\omega)$ is an instrument factor obtained by instrument calibration, $\mathrm{z}_{\mathrm{s}}$ and $\mathrm{z}_{\mathrm{g}}$ are acoustic impedance factors of the suspension and the instrumentation respectively, $\phi$ is the particle volume fraction, $\rho_{\mathrm{p}}$ and $\rho$ are the density of the particles and the solvent respectively and $\mu_{\mathrm{D}}$ is the dynamic mobility. The inertia factor $\mathrm{G}(\mathrm{a})$ needed to calculate the zeta potential $\zeta$ from dynamic mobility (equation 2 ) is determined by the ZetaProbe software.

$$
\mu_{D}=\varepsilon \zeta / \eta \cdot G(a)
$$

The instrument measures the dynamic mobility over a range of frequencies and the software finds the particle size distribution and zeta potential from the best fit between the 
measured and theoretical dynamic mobility spectra [20]. Control measurements were performed observing the effect of the temperature, the stirring speed and variations over sample duplicates. A maximum uncertainty of $2 \mathrm{mV}$ was estimated considering average values accounting for all these parameters.

Sedimentation studies were performed using a Turbiscan Lab (Formulaction, France) using the Turbisoft software version 2.1.0.52.

Samples were loaded into vials with $ø \approx 27 \mathrm{~mm}$ and to a height of approximately $40 \mathrm{~mm}$ (packing was normalized with the starting height) and measurements are performed for every $40 \mu \mathrm{m}$ along the entire height. 5 initials scans were made in order to ensure a nice background measurement and subsequently data was acquired once every 30 minutes the following 24 hours in order to obtain a good estimation of the initial sedimentation speed. After the first 24 hours data was recorded approximately twice a week or as needed until final packing was achieved. The studies were carried out in a thermostated lab. with an almost constant temperature of $21^{\circ} \mathrm{C} \pm 1^{\circ} \mathrm{C}$ ensuring little variation in sample viscosity. The equipment is set up with a laser operating at $880 \mathrm{~nm}$ and is equipped with two detectors. A transmission detector for non-opaque samples placed opposite to the laser and a backscatter detector at $45^{\circ}$ for opaque samples. As the volume fraction of the investigated samples are significantly above $\approx 0.1 \mathrm{vol} \%$ data from the backscatter detector was used for calculation of sedimentation rates etc. Data treatment was performed from the top of the glass bottom to the bottom of the liquid meniscus in order to avoid unnecessary reflections at e.g. liquid to air interfaces. As sensitivity level, a standard relative threshold of $50 \%$ was used for following the sedimentation front. 
A major advantage of the applied techniques for measuring sedimentation speed and zeta potential is the ability to measure on suspensions without dilution, i.e. conditions that resemble the real interactions in high ceramic solid load slurries used for tape casting.

\section{Results}

\subsection{YSZ powder in ethanol}

The particle size distribution (PSD) of YSZ powder suspended in ethanol was recorded as a function of time in combination with the evolution of the zeta potential of the material. No dispersants was added and the suspension was kept under mild mixing conditions. The curves representing PSD are collected in Figure 1, while zeta potential values are plotted in Figure 2. Figure 1 shows that after 6 hours the YSZ powder has a broad monomodal particle size distribution centered around 40-50 $\mu \mathrm{m}$. By rolling in ethanol, some of these big agglomerates break down to smaller size and form a more complex PSD, where agglomerates with diameter between $60 \mathrm{~nm}$ and $6 \mu \mathrm{m}$ increase in volume percent with time. Their re-agglomeration is increasingly impeded from the positive surface potential, which develops with time as seen in Figure 2. Even though the ZetaProbe conductivity measurements are not accurate below $50 \mu \mathrm{S} / \mathrm{cm}$, the conductivity steadiness indicates that the suspension ionic strength does not change with time, but it is significantly higher than the conductivity of the solvent alone (which is below the lower limit of conductivity for the instrument and therefore not reported here).

A much larger and faster effect in the reduction of agglomerates is obtained by ball milling, as visible in Figure 1 (graph marked with starts). The sample milled for 2 days does not show agglomerates bigger than $20 \mu \mathrm{m}$, has only 2 small residual populations centered at 4- 
$5 \mu \mathrm{m}$ and $15 \mu \mathrm{m}$ while the majority of agglomerates ranges between $40 \mathrm{~nm}$ and $3 \mu \mathrm{m}$. The positive value of zeta potential is also reached faster under milling conditions as indicated by the single point at $37 \mathrm{mV}$ in Figure 2.

\subsection{Comparison between dispersants}

The particle size distribution in the YSZ suspensions milled with PVP, PEI and PVBAcetal are plotted in Figures 3, 4 and 5 respectively. The reference curve, corresponding to the ceramic powder ball milled in ethanol without dispersant, is also reported in the 3 figures for easier comparison. The optimum amount of PEI and PVP corresponds to the range of 1$2.5 \mathrm{dwb} \%$, corresponding to $0.7-1.8 \mathrm{mg} / \mathrm{m}^{2}$. Further addition of these dispersants does not provide any further reduction in particle size. Therefore not all the measurements at higher dispersant concentration are reported in the graphs. On the other hand it was not possible to eliminate the 3-6 $\mu \mathrm{m}$ agglomerates using PVBAcetal, even increasing its amount in suspension up to $16 \mathrm{dwb} \%$. Figure 6 puts in comparison the best results achieved with PVP (2.5 dwb\%), PEI (1.0 dwb\%) and the sample ball milled without dispersant for 2 days.

The milling kinetic of YSZ with the PEI dispersant was investigated by observing the particle size evolution as a function of milling time. The results are collected in Table 1 for a suspension containing $2 \mathrm{dwb} \%$ of PEI.

\begin{tabular}{|c|c|c|c|c|c|}
\hline \multirow{2}{*}{$\begin{array}{c}\text { Milling time } \\
\text { (days) }\end{array}$} & \multicolumn{5}{|c|}{ Particle size distribution $(\boldsymbol{\mu m})$} \\
\cline { 2 - 6 } & $\mathbf{D}_{\mathbf{1 0}}$ & $\mathbf{D}_{\mathbf{2 5}}$ & $\mathbf{D}_{\mathbf{5 0}}$ & $\mathbf{D}_{\mathbf{7 5}}$ & $\mathbf{D}_{\mathbf{9 0}}$ \\
\hline 0 & 36 & 49 & 68 & 89 & 108 \\
\hline 1 & 0.1 & 0.1 & 0.2 & 1.0 & 1.6 \\
\hline 2 & 0.1 & 0.1 & 0.2 & 0.8 & 1.3 \\
\hline 3 & 0.1 & 0.1 & 0.2 & 0.6 & 1.1 \\
\hline 4 & 0.1 & 0.1 & 0.2 & 0.2 & 0.9 \\
\hline 7 & 0.1 & 0.1 & 0.2 & 14 & 26 \\
\hline
\end{tabular}

Table 1. Milling kinetic of YSZ suspension in ethanol containing $2 d w b \%$ PEI dispersant. 
The initial point in Table 1 corresponds to a freshly prepared dispersion, after 2 hours of mixing without milling beads. It appears that the major step in the comminution process is achieved during the first 24 hours milling, while in the following 3 days only $\mathrm{D}_{75}$ and $\mathrm{D}_{90}$ continues to decrease. For longer milling time, $\mathrm{D}_{75}$ and $\mathrm{D}_{90}$ rise again pointing out to the reformation of large aggregates. On the other hand another portion of the same YSZ suspension, set to roll slowly without milling beads for 12 days after the initial 4 days of milling, shows very good stability in these conditions and no sign of re-agglomeration (Figure 7).

PSD was also measured on high solid load suspension prepared for tape casting and stabilized with PEI and PVP. The results are plot in Figure 8 and are completely comparable to the suspension used for the screening process.

A rheological study was also attempted on these suspensions and the flow curves were collected on the samples with and without addition of dispersants. Because of the low samples' viscosity, the measurements were performed on the sensitivity limit of the rheometer and the data are not reported here. In spite of the high level of noise, a clear difference in viscosity is visible between the suspensions containing polymeric dispersants and suspension without a stabilizing agent. In the presence of the dispersant the flow curves have almost Newtonian behavior above shear of $1 \mathrm{~s}-1$ and viscosity of few $\mathrm{mPa} \cdot \mathrm{s}$. Suspensions of YSZ in ethanol display shear thinning behavior and considerably higher viscosity, confirming the presence of larger and more numerous aggregates, as seen from PSD results. 
Figure 9a shows the sedimentation rate as a function of dispersant concentration in the YSZ suspensions for the three different dispersing polymers and for the sample of YSZ in ethanol after 2 days milling. The initial sedimentation rate was calculated for each suspension by following the position of the clarifying front as a function of time by measuring the intensity of the back-scattered light and finding the slope at early times corresponding to the regime with free falling particles. Similarly, the position of the settling front at the bottom of the glass jar was followed by back-scattering with time. The ratio between the height of the YSZ cake at equilibrium and the initial height of the suspension is plotted as a function of dispersant concentration in Figure 9b. All the polymers clearly reduce the sedimentation speed when they are added to the suspensions in concentration above $0.5-1.0 \mathrm{dwb} \%$. The height of the solidification front is considerably reduced (about $60 \%$ ) by all dispersants in the range of $1-4 \mathrm{dwb} \%$. It appears that higher concentration of PEI produces even higher cake density, which is an indication of better packing. As mentioned above, all the viscosity measurements performed on YSZ suspensions show comparable values irrespectively of the dispersant considered (PVP and PEI) and its concentration $(2-10 \mathrm{dwb} \%)$ within the pretty large error bar in the low viscosity range. In any case all the values were below $7 \mathrm{mPa} \cdot \mathrm{s}$ at shear rate $1 \mathrm{~s}^{-1}$. The YSZ suspensions without dispersing agent have viscosity around $150 \mathrm{mPa} \cdot \mathrm{s}$ in the same shear rate range. This observation insures that the slower sedimentation rate recorded with all the dispersant is not affected by the increased suspension viscosity, but rather due to better de-agglomeration and smaller agglomerate size.

The zeta potential values of the YSZ suspensions with different dispersants milled for 2 days in ethanol as a function of the dispersant concentration are reported in Figure 10. The 
YSZ suspension is characterized by a positive charge in ethanol, indicating basic properties of the composite oxide. When PVP or PVBAcetal are present in the solution the zeta potential decreases monotonically with the polymer amount. On the other hand, with PEI the potential curve shows a maximum potential for low amount of dispersant and a monotonic decrement similar to the other two dispersing agents for larger concentrations of the polymers.

\subsection{Microstructure on sintered tapes}

The thin films produced by tape casting were characterized with SEM after sintering. The micrographs in cross section of the tapes produced with PVP and PEI dispersant are reported in Figure 11 a) and 11 b) respectively. Figures 12 a) and b) show the micrographs at high resolution for the same tapes in cross section produced using PVP and PEI respectively, where the samples have been thermally etched such that the grain boundaries are visible. The samples show similar packing and grain sizes.

\section{Discussion}

As pointed out in [21], a certain degree of electrolyte dissociation is possible in polar organic media, such as the lower alcohols having moderate dielectric constant. Ethanol is a polar, protic solvent with very weak acidic properties $(\mathrm{pKa}=15.9)$, auto-protolysis constant $\mathrm{K}_{\mathrm{EtOH}}$ of 19.1 [22] and dielectric constant of 24.3 [23]. As shown in Figure 2, at equilibrium the 3YSZ powder acquires a considerable positive potential in ethanol, showing that electrostatic stabilization represents an important contribution also in this non-aqueous system. At the same time, the increment in conductivity after powder addition proves an increment of ionic concentration and a certain degree of electrolyte dissociation. Even 
though ethanol has lower polarity and dielectric constant than water, the stability properties can still be interpreted according to the classical Derjaguin-Landau-Verwey-Overbeek (DLVO) theory [21 and 24], where the total potential energy between particles is described by electrostatic repulsion and London-Van der Waals attraction forces [25].

Since the powder was not calcined, but kept at $120^{\circ} \mathrm{C}$ prior to use, the presence of partially hydrated species on the ceramic surface in form of $\mathrm{Y}-\mathrm{OH}$ and/or $\mathrm{Zr}-\mathrm{OH}$ is expected. The charging mechanism of the surface is normally described by the adsorption of protons from the solvent (ethanol or atmospheric water dissolved in it) onto the ceramic surface, following the schematic equations (1) and (2), where the powder surface behaves as a base:

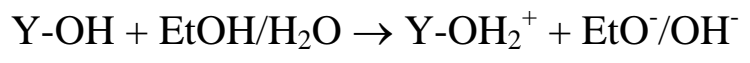

$$
\begin{aligned}
& \mathrm{Zr}-\mathrm{OH}+\mathrm{EtOH} / \mathrm{H}_{2} \mathrm{O} \rightarrow \mathrm{Zr}-\mathrm{OH}_{2}{ }^{+}+\mathrm{EtO}^{-} / \mathrm{OH}^{-}
\end{aligned}
$$

Based on the isoelectric point values for $\mathrm{Y}_{2} \mathrm{O}_{3}$ and $\mathrm{ZrO}_{2}$ in water (IEP of $\mathrm{Y}_{2} \mathrm{O}_{3}=8.8$; IEP of $\mathrm{ZrO}_{2}=6.5$ ) [26], $\mathrm{Y}_{2} \mathrm{O}_{3}$ is the strongest base in the $\mathrm{YSZ}$ compound. Therefore, it is $\mathrm{Y}_{2} \mathrm{O}_{3}$ that mainly confers the basic properties to the powder surface, especially assuming a certain degree of $\mathrm{Y}_{2} \mathrm{O}_{3}$ enrichment at the particle surface.

The PSD graphs collected during rolling YSZ suspension in Figure 1 show that some of the big agglomerates can be easily broken by mild mixing energy. As the intensity of the large signal (initial population between 20 and $200 \mu \mathrm{m}$ ) decreases with time while the volume $\%$ of the smaller agglomerates increases, we can conclude that the surface charge (which also develops with time) ensures enough electrostatic repulsion to avoid aggregation by Van der Waals attraction forces and gives significant contribution to the suspension stabilization. As expected, the energy given by ball milling breaks the large agglomerates much faster 
and the population between 20 and $200 \mu \mathrm{m}$ almost completely disappears after 2 days. The sedimentation data recorded on the suspensions without dispersants are represented as squares in Figure 9 and indicate a sedimentation rate slightly below $1 \mathrm{~mm} / \mathrm{h}$. On the base of the Stoke's law, agglomerates larger than $10 \mu \mathrm{m}$ will not be tracked even by the initial laser scans as they sediment too fast. For $1-\mu \mathrm{m}$ agglomerates, the settling velocity is smaller and they will sediment in a few hours. The measured sedimentation speed of $1 \mathrm{~mm} / \mathrm{h}$ corresponds well with the major PSD peak just above $100 \mathrm{~nm}$ seen by light scattering. The contribution of the dispersants PEI, PVB and PVP (Figures 3-6) is to modify the particle size distribution further, especially the proportions of the particle populations below $2 \mu \mathrm{m}$. Especially in the presence of PEI and PVP, the volume percent of the $100 \mathrm{~nm}$ population exceeds the 1-2 $\mu$ m-population, and the aggregates larger than $2 \mu \mathrm{m}$ completely disappear. All the dispersants allow slower sedimentation and better packing as shown in Figure 9. Even though zeta potential reaches values of $35-38 \mathrm{mV}$ in the YSZ/ethanol system, the repulsion forces and the Debye length are not large enough to provide full stability to sub-micron agglomerates. Similarly, experiments and calculations performed on alumina suspensions in ethanol show that full stability by electrostatic repulsion was obtained only for $|\zeta|>43 \mathrm{mV}[21]$ and was obtained by acid addition to the suspension. More details about the stabilization effects and specific properties of the three dispersing polymers are output of the single different analysis techniques. PSD results say that the amount of PEI and PVP required to obtain the smallest PSD are comparable. PVBAcetal does not provide the same degree of de-agglomeration up to $16 \mathrm{dwb} \%$. In agreement with stability studies published for PVB dispersants [16], PVBAcetal might have lower affinity 
for the ceramic surface and be easily displaced by the solvent. For this reason, higher concentration of polymer in suspension is required for covering the ceramic particles surface. Therefore the use of PVP and PEI would be preferable for their higher affinity for the YSZ particles.

The shape of the curve representing zeta potential vs. dispersant concentration in Figure 10, gives indications about the stabilization mechanism. PVP and PVBAcetal show the typical behaviour of steric stabilization, where the addition of dispersant makes the hydrodynamic radius larger and the potential at the shear plane becomes lower. In the case of PEI the maximum in the potential indicates a more complex mechanism, where low dispersant concentration increases the particle charge even further. At PEI concentration higher than 1 $\mathrm{dwb} \%$ the potential decay resembles that observed for steric stabilization, but the charge measured for optimum dispersant amount is much larger than for the other 2 polymers, introducing an electro-steric stabilizing repulsion. A clear explanation for this behavior was not found yet and could be subject for further investigations. At our knowledge the PEI dissociation constant (or its charge) in ethanol are not available in literature. We found instead calculated and experimental values of aliphatic secondary and tertiary ammines in ethanol [27]. For instance $\mathrm{Et}_{2} \mathrm{NH}_{2}{ }^{+}$has $\mathrm{pKa}$ (calculated) $=10.7$ and $\mathrm{pKa}$ (experimental) $=10.97 . \mathrm{Bu}_{3} \mathrm{NH}^{+}$has $\mathrm{pKa}($ calculated $)=9.3$ and $\mathrm{pKa}($ experimental $)=10.23$. Using the ethanol autoprotolysis constant of $10^{-19}$, the resulting $\mathrm{pKb}$ are close to 8 for the secondary ammine and close to 9 for the ternary ammine. Based on such a low basicity and the weak ethanol acidity, we expect that PEI may carry only a very small positive charge. The nature of interactions between PEI and the ceramic surface might be based on hydrogen bonds formation between $\mathrm{ZrOH}$ groups on the ceramic surface and $\mathrm{C}-\mathrm{N}$ groups on the PEI 
polymer, as explained in [28] for zirconia powder in water below the IEP (where the ceramic particles have positive zeta potential and the PEI is positively charged).

The sedimentation measurements show that the sedimentation speed for the three dispersants is very similar between 2 and $8 \mathrm{dwb} \%$. The heights of the cake deposited on the vial bottom are also comparable for the 3 samples when the dispersant concentration ranges between 1 and $5 \mathrm{dwb} \%$. This information confirms a general good correlation between small particle size, low sedimentation speed and good compaction of the solid, which can be expected also in the green body after tape casting.

Significant differences between dispersants can be seen when the dispersant concentration is below the optimal value. Especially for low PEI concentration, the PSD appears very large: Probably the polymer works as a bridge between particles and induces flocculation. The flocculation induced by PVP is not as evident as seen with PEI. Lower sedimentation speed for low concentration of PEI compared to the other 2 polymers in spite of larger PSD, could be due to the formation of a 3-dimentional structure between polymer and ceramic particles.

On the bases of the good performance in the stability tests, PEI and PVP were chosen for tape casting with optimized amount of dispersant. YSZ suspensions prepared with PVP and PEI at high solid load could be milled to optimal particle size (Figure 8). This proved that our selection method performed with $28.6 \mathrm{wt} \%$ ceramic solid load is appropriate for investigating suspension stability and it gives the opportunity to limit excessive material consume for large series of samples. Leak tests on the sintered ceramic prove that the final product has only closed porosity, as also verified by SEM images, which confirm good densification. After thermal etching, the high-resolution SEM images show that the dense 
layers are multi-grained, with uniform grain size around 200-300 nm. In reference [29] a remarkably fine-grained, high-density sintered sample is obtained using $12 \mathrm{~nm}$ YSZ crystallites pressed into pellets. A similar fine and dense microstructure characterized both the samples produced in our work with PEI and PVP. Therefore the high-resolution SEM analysis strongly indicates that the two dispersants are equally good.

\section{Conclusions}

This work describes the dispersion capabilities of some stabilizing agents for YSZ in ethanol: The molecules considered are the polymers PVP, PEI and PVBAcetal. The analytical tools used allow studies in suspension with high ceramic solid load, relevant for slurries suitable for tape casting applications. It was observed that the ceramic powder develops a positive potential after some days of equilibration in ethanol. The positive charge formed on the particle surface produces some electrostatic repulsion and helps comminution by ball milling, but it is not enough for complete stabilization of the powder. It was proven that the addition of polymeric dispersants ensures better stability, by observation of smaller particle size distribution, slower sedimentation rate and better packing. The biggest effect of the dispersants is given in the low range of particle size, and largely reduces the 1-2 $\mu$ m population. Zeta potential measurements confirm a mechanism of steric stabilization for PVP and PVBAcetal and point out to an electro-steric mechanism with PEI. Even though PVBAcetal reduces sedimentation rate and particle size distribution, it shows lower affinity to the YSZ powder compared to the other two polymers. On the bases of the good performance, PEI and PVP were chosen for tape casting with optimized 
amount of dispersant. After sintering the thin films produced proved to be leak tight and SEM images show good densification and only closed porosity.

The presence in the suspensions of 2 populations with different particle size, in any case larger than the crystallite size, shows that the energy required for further comminution is not developed with the ball milling procedure, but the final PSD achieved is suitable for tape casting and for densification during sintering.

\section{Acknowledgments}

This work was carried out within the SymSOEC project, supported by the Department of Energy Conversion and Storage, DTU. The authors would like to express their appreciation to Marianne Nielsen and Henrik Paulsen for the accuracy in the tape casting procedure and quality in technical support in sample preparation. The authors are grateful to Karl Tor Sune Thydén for the high resolution SEM images; to Karen Brodersen and Mads GudikSørensen for useful discussion and suggestions in the dispersant choice.

\section{References}

[1] N.Q. Minh, T. Takahashi, Science and technology of ceramic fuel cells, Elsevier Science B.V., Amsterdam (1995).

[2] A. Nakajo, J. Kuebler, A. Faes, U. F. Vogt, H. J. Schindler, L.-K. Chiang, S. Modena, J. Van Herle, T. Hocker, Compilation of mechanical properties for the structural analysis of solid oxide fuel cell stacks. Constitutive materials of anode-supported cells, Ceramic International 38 (2012) 3907-3927. 
[3] J. S. Reed, Principles of ceramics processing, John Wiley \& Sons, Inc., New York (1995).

[4] Raeder, H. Simon, C. Chartier, T. Toftegaard, H.L. Tape casting of zirconia for ion conducting membranes: A study of dispersants, Journal of the European Ceramic Society 13 (1994) 485-491.

[5] R. Moreno, The role of slip additives in tape-casting technology: Part I - Solvents and dispersants, American Ceramic Society Bulletin 71 (1992) 1521-1531.

[6] E.P. Hyatt Making Thin, Flat ceramics- A Review, Ceramic Bulletin 65 No. 4 (1986) 637-638.

[7] H. Amorin, I. Santacruz, J. Holc, M. P. Thi, M. Kosec, R. Moreno, M. Algueró, Tapecasting performance of ethanol slurries for the processing of textured PMN-PT ceramics from nanocrystalline powder J. Am. Ceram. Soc. 92 (2009) 996-1001.

[8] G. Blugan, K. Morawa, S. Koebel, T. Graule, J. Kuebler, Development of a tape casting process for making thin layers of $\mathrm{Si}_{3} \mathrm{~N}_{4}$ and $\mathrm{Si}_{3} \mathrm{~N}_{4}+\mathrm{TiN}$ Journal of the European Ceramic Society 27 (2007) 4789-4795.

[9] T. Klemens $\varnothing$, M. Menon and S. Ramousse, Low Toxicity Binder Systems for Tape Cast $\mathrm{Ce}_{0.9} \mathrm{Gd}_{0.1} \mathrm{O}_{1.95}$ Laminates, Ceramics International 36 (2010) 773-780.

[10] S. B. Reddy, P. P. Singh, Effect of type of solvent and dispersant on NANO PZT powder dispersion for tape casting slurry, Journal of materials Science, 37 (2002) 929-934 [11] K. K. Hansen, M. Menon, J. Knudsen, N. Bonanos, M. Mogensen, The Effect of a CGO Barrier Layer on the Performance of LSM/YSZ SOFC Cathodes, J. Electrochem. Soc. 157 (2010) B309-B313. 
[12] Meng Shan, Xiaojian Mao, Jian Zhang, Shiwei Wang, Electrophoretic shaping of submicron alumina in ethanol, Ceramic International 35 (2009) 1855-1861.

[13] A.M. Popa, J. Vleugels, J. Vermant, O. Van der Biest, Influence of ammonium salt of poly-methacrylic acid and butylamine addition on the viscosity and electrophoretic deposition behavior of ethanol-based powder suspensions, Colloids and Surface A: Physicochemical Engineering Aspects 267 1-3 (2005) 74-78.

[14] Wenjea J. Tseng, Chun-Liang Lin, Effect of polyvinyl butyral on the rheological properties of BaTiO3 powder in ethanol-isopropanol mixtures, Material Letters 57 (2002) $223-228$.

[15] Zhang Jingxian, Jiang Donglianga, Lars Weisenselb, Peter Greil, Binary solvent mixture for tape casting of TiO2 sheets, Journal of European Ceramic Society 24 (2004) $147-155$.

[16] S. Bhattacharjee, M. K. Paria, H. S. Maiti, Polyvinyl butyral as a dispersant for barium titanate in a non-aqueous suspension, Journal of Materials Science, 28 (1993) 6490-6495. [17] Reference given by the supplier Kuraray: http://www.kuraray.eu/fileadmin/Downloads/pvb/Mowital_pioloform_broschuere_2013_1 7042013_low_quality_secured.pdf.

[18] S. Ramousse, M. Menon, K. Brodersen, J. Knudsen, U. Rahbek, P.H. Larsen, Manufacturing of Anode-Supported SOFC's: Processing Parameters and their Influence, ECS Transactions 7 (2007) 317-327.

[19] C.M. Keck, R.H. Müller, Size analysis of submicron particles by laser diffractometry$90 \%$ of the published measurements are false, International Journal of Pharmaceutics 355 (2008) 150-163. 
[20] R.W. O’Brien, A. Jones, W.N. Rowlands, A new formula for the dynamic mobility in a concentrated Colloid, Colloids and Surfaces A: Physicochem. Eng. Aspects 218 (2003) 89-101.

[21] Gonghou Wang, Partho Sarkar, Patrick S. Nicholson, Influence of acidity on the electrostatic stability of alumina suspensions in ethanol, Journal of American Ceramic Society 80 (1997) 965-972.

[22] Bates R.G. Determination of $\mathrm{pH}$. Theory and Practice, $2^{\text {nd }}$ edition, Wiley, New York, 1973.

[23] R.C. Weast (ed.), Handbook of Chemistry and Physics; $71^{\text {st }}$ ed. CRC Press, 1990. [24] J Lyklema, Principles of interactions in non-aqueous electrolyte solutions, Current Opinion in Colloid \& Interface Science 18 (2013) 116-128.

[25] E.J.W. Vervey and J.Th.G. Overbeek, Theory of the Stability of Lyophobic Colloids, Elsevier, Amsterdam, the Nederlands, 1948.

[26] Masahiro Kagawa, Mamoru Omori, Yasuhiko Syono, Yoko Imamura, Shinnosuke Usui, Surface characterization of zirconia after reaction with water, J. Am. Ceram. Soc. 70 [9] (1987) C212-C213.

[27] Yu. E. Zevatskii, D.O. Ruzanov, and D.V. Samoilov "Experimental Testing of the Calculation Results of the Dissociation Constant of Organic Compounds in Nonaqueous Media” Russian Journal of of General Chemistry 79 [9] (2009) 1533-1537.

[28] J. Wang and L. Gao "Adsorption of Polyethylenimine on Nanosized Zirconia Particles in Aqueous Suspensions” Journal of Colloids and Interface Science 216 (1999) 436-439. [29] W H Rhodes “Agglomerate and Particle Size Effects on Sintering Yttria-Stabilized Zirconia” J. Am. Ceram. Soc. 64 [1] (1981) 19-22. 


\section{Figure captions}

Figure 1. PSD of YSZ suspensions in ethanol without dispersant as a function of time in mild mixing conditions and milling with ceramic beads.

Figure 2. Zeta potential and conductivity of YSZ suspensions in ethanol without dispersant as a function of time in mild mixing conditions and milling with ceramic beads.

Figure 3. PSD of YSZ suspensions in ethanol with PVP at different dispersant concentrations.

Figure 4. PSD of YSZ suspensions in ethanol with PEI at different dispersant concentrations.

Figure 5. PSD of YSZ suspensions in ethanol with PVBAcetal at different dispersant concentrations.

Figure 6. PSD of YSZ suspensions in ethanol comparing different dispersing agents: PVP, PEI at optimal dispersant concentration.

Figure 7. PSD of YSZ suspensions in ethanol containing $2 \mathrm{dwb} \%$ PEI. The measurement was performed after 4 days milling and repeated after 12 days rolling without milling beads.

Figure 8. PSD of YSZ suspensions in ethanol containing $2 \mathrm{dwb} \%$ PEI and $2.5 \mathrm{dwb} \%$ PVP after 3 days milling with $66 \mathrm{w} / \mathrm{w} \%$ ceramic solid load.

Figure 9. Sedimentation rate (a) and sediment height (b) of YSZ suspensions in ethanol comparing different dispersing agents and different concentrations.

Figure 10. Zeta potential curves as a function of dispersant concentration for the 3 different 
dispersants.

Figure 11. SEM micrographs of YSZ sintered tapes in cross section, produced with PVP (a) and PEI (b) as dispersing agents in the slurry formulation for tape casting.

Figure 12. High resolution SEM micrographs of YSZ sintered tapes in cross section, produced with PVP (a) and PEI (b) as dispersing agents in the slurry formulation for tape casting. 


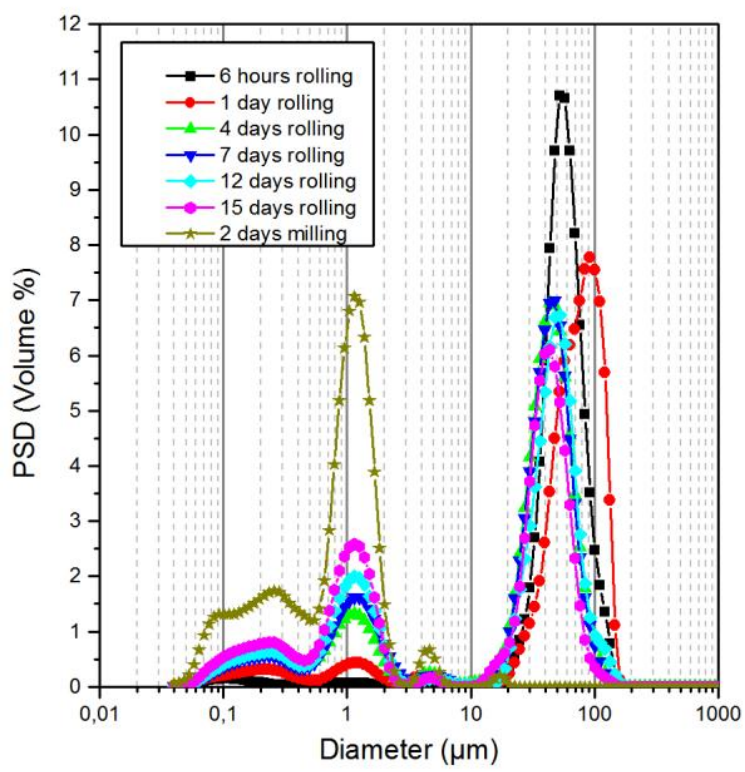




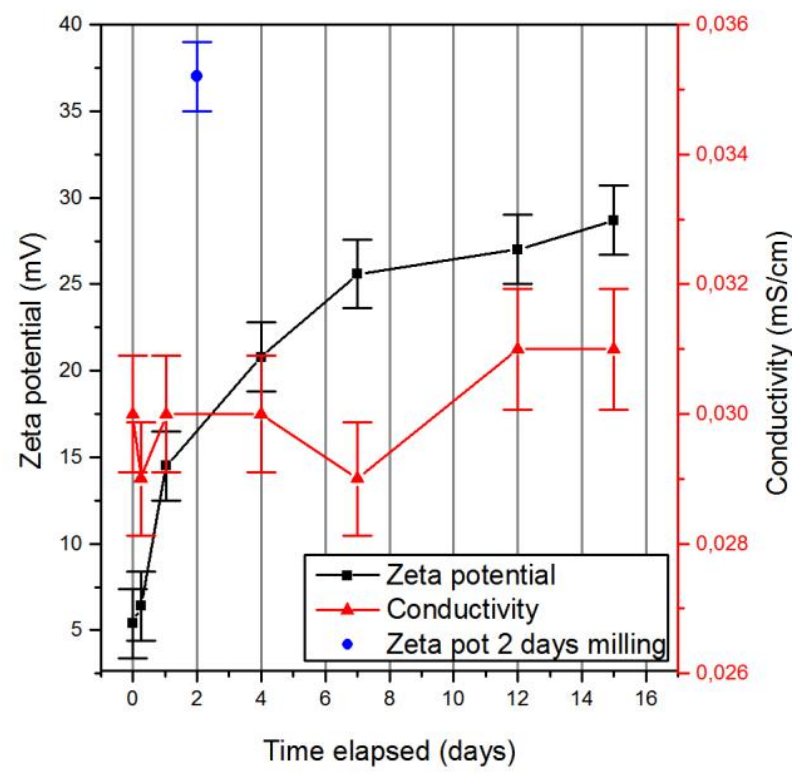




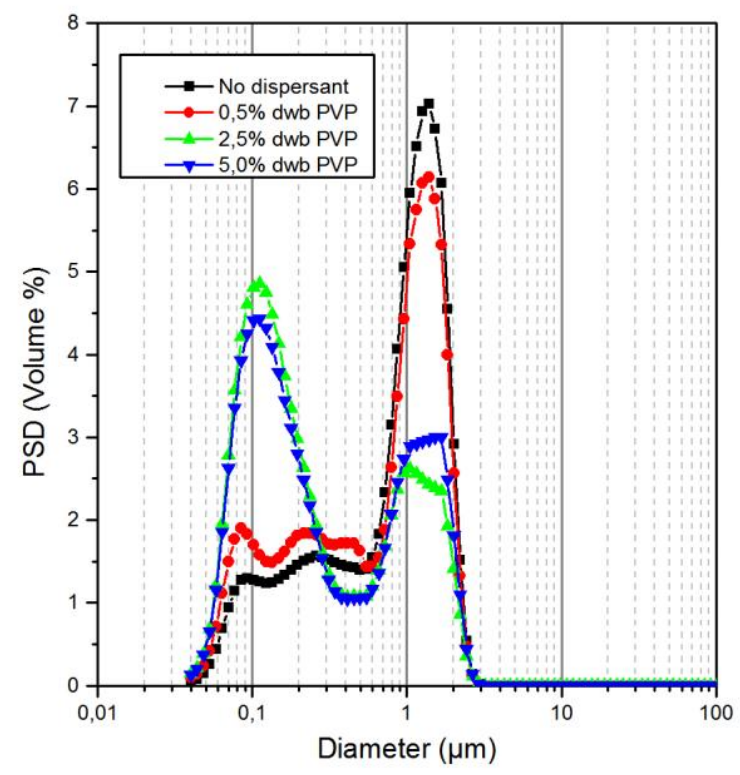




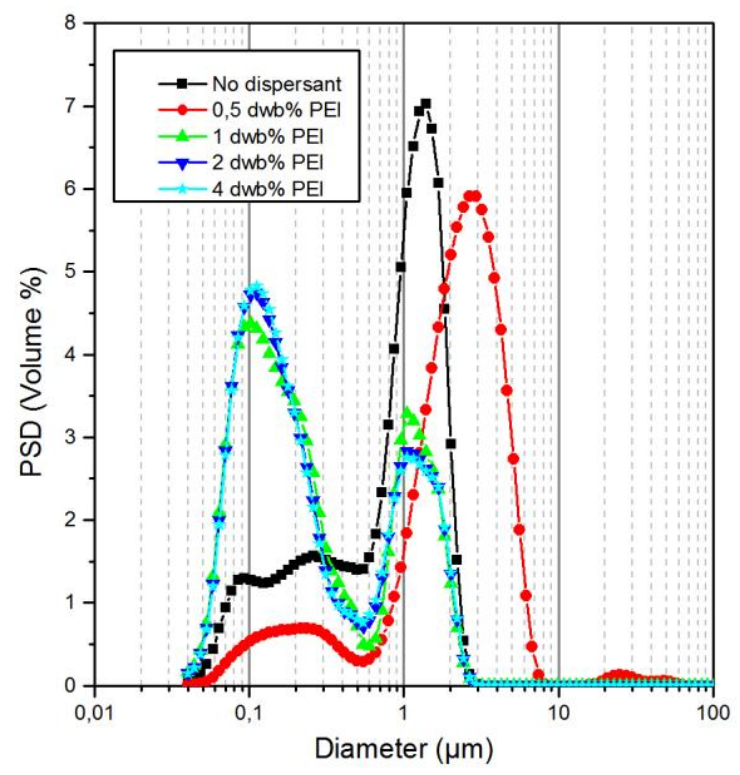




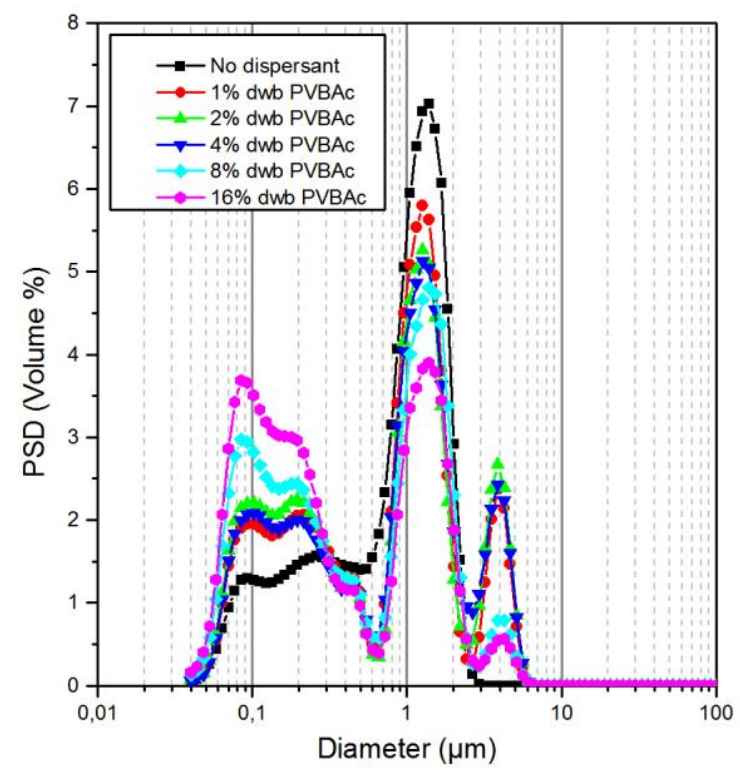




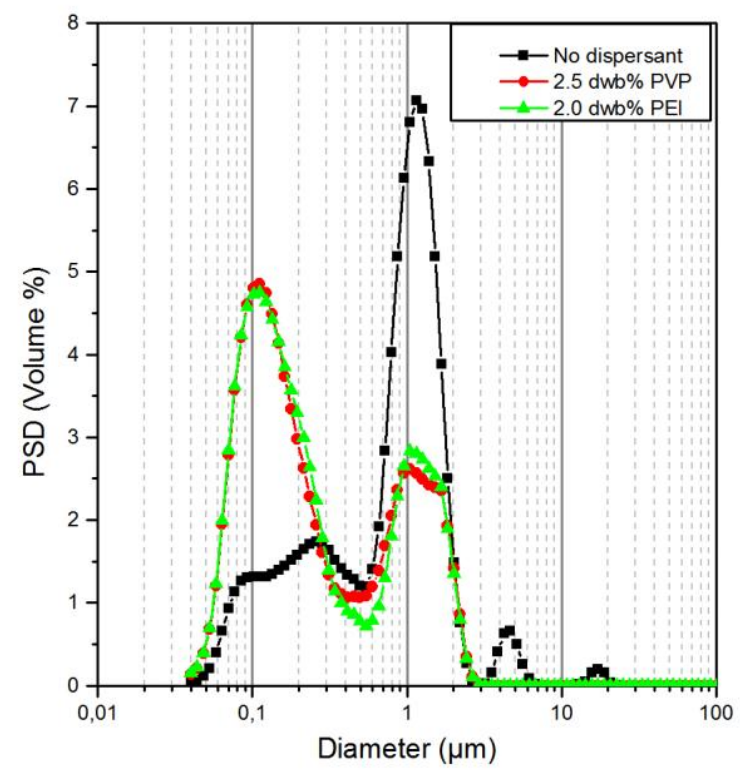




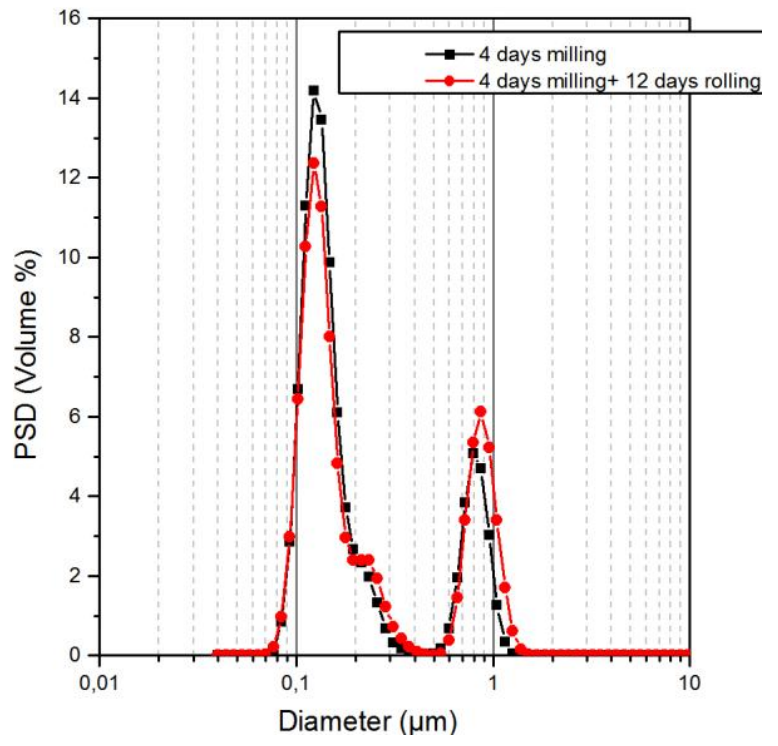




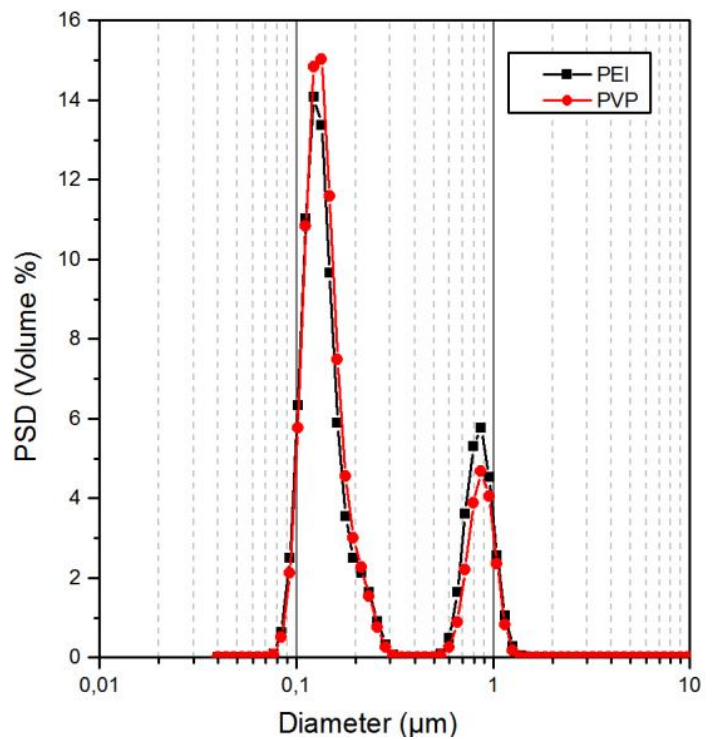




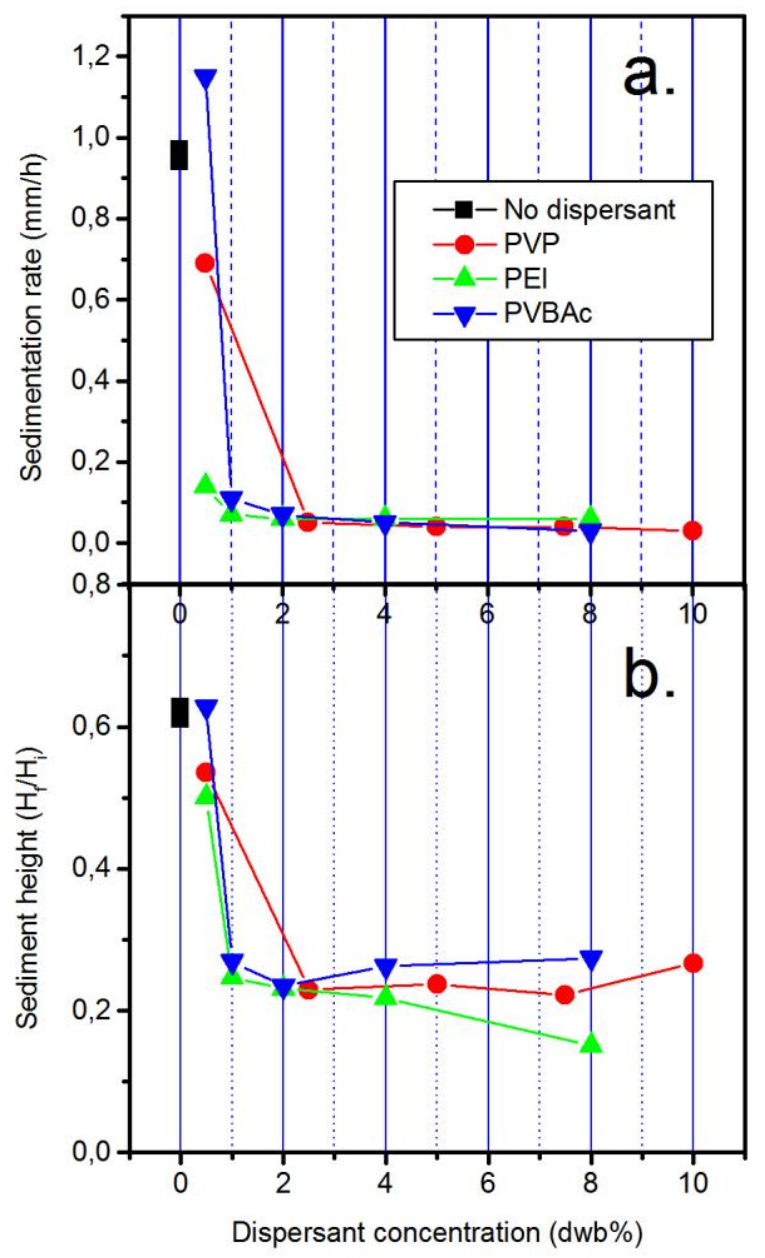




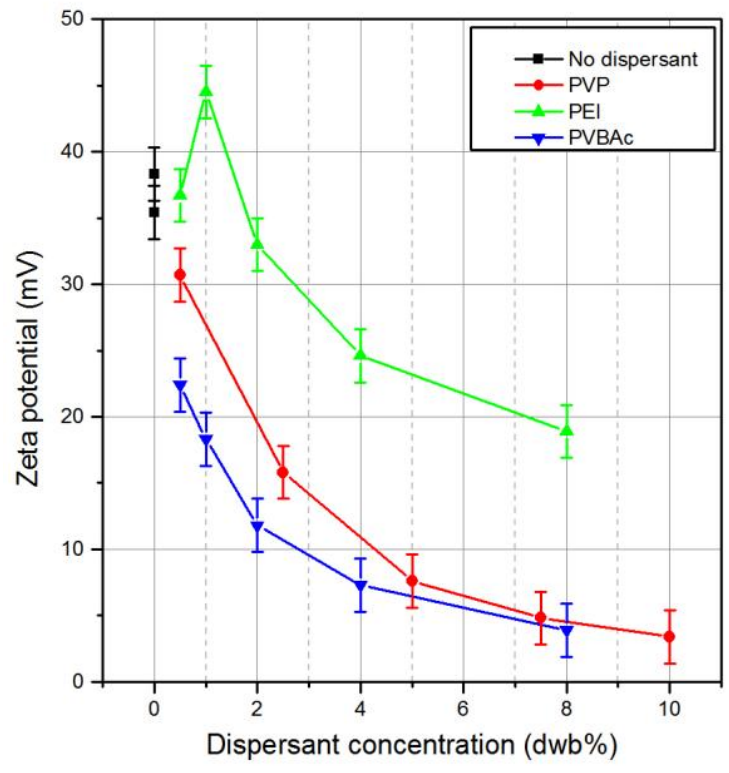




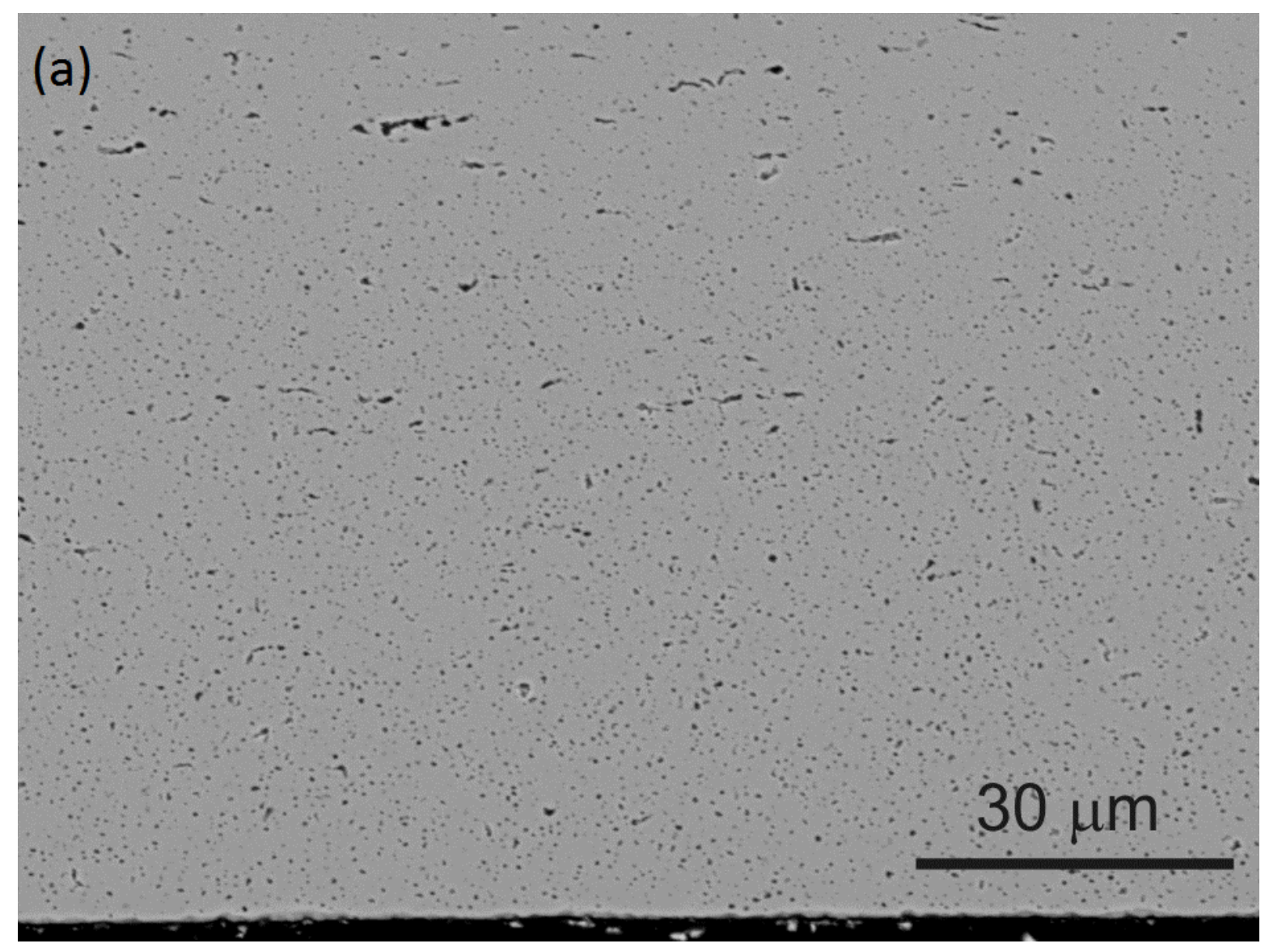




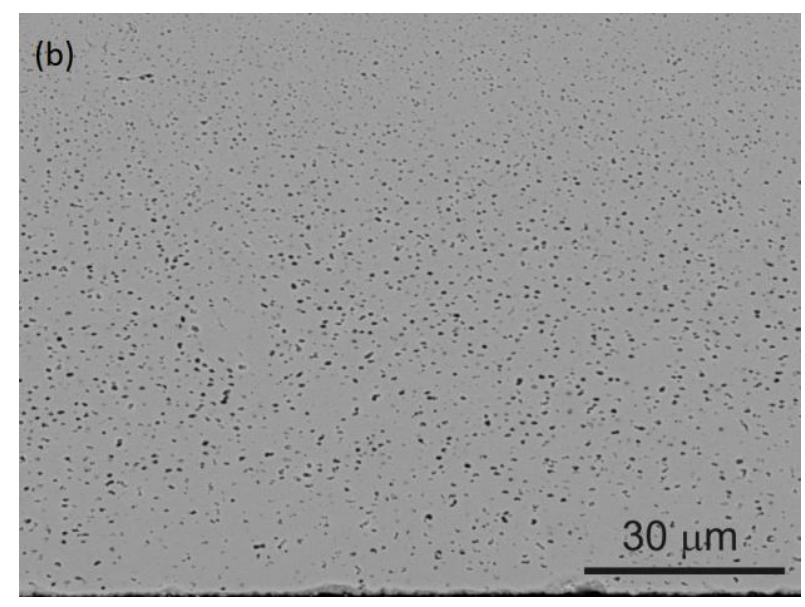




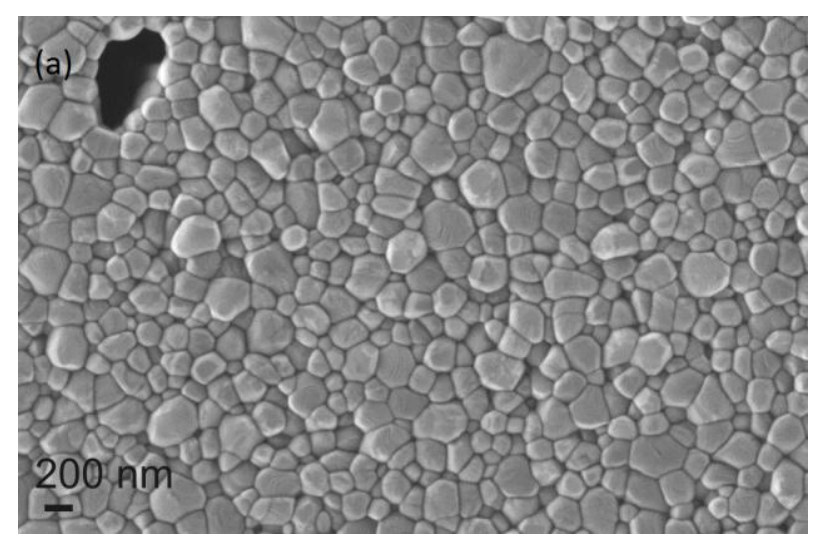




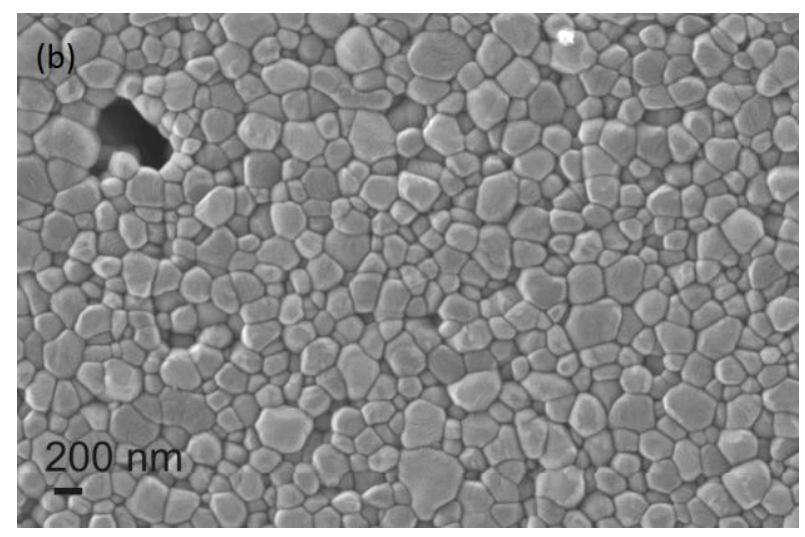

